\title{
Tumor suppressor gene RBM5 delivered by attenuated Salmonella inhibits lung adenocarcinoma through diverse apoptotic signaling pathways
}

Chen Shao', Baoxue Yang ${ }^{4}$, Lijing Zhao ${ }^{2,3}$, Song Wang ${ }^{5}$, Jie Zhang ${ }^{1}$ and Ke Wang ${ }^{1 *}$

\begin{abstract}
Background: RBM5 (RNA-binding motif protein 5, also named H37/LUCA-15) gene from chromosome 3p21.3 has been demonstrated to be a tumor suppressor. Current researches in vitro confirm that RBM5 can suppress the growth of lung adenocarcinoma cells by inducing apoptosis. There is still no effective model in vivo, however, that thoroughly investigates the effect and molecular mechanism of RBM5 on lung adenocarcinoma.

Method: We established the transplanted tumor model on BALB/c nude mice using the A549 cell line. The mice were treated with the recombinant plasmids carried by attenuated Salmonella to induce the overexpression of RBM5 in tumor tissues. RBM5 overexpression was confirmed by immunohistochemistry staining. H\&E staining was performed to observe the histological performance on plasmids-treated A549 xenografts. Apoptosis was assessed by TUNEL staining with a TUNEL detection kit. Apoptosis-regulated genes were detected by Western blot.
\end{abstract}

Results: We successful established the lung adenocarcinoma animal model in vivo. The growth of tumor xenografts was significantly retarded on the mice treated with pCDNA3.1-RBM5 carried by attenuated Salmonella compared to that on mice treated with pcDNA3.1. Overexpression of RBM5 enhanced the apoptosis in tumor xenografts. Furthermore, the expression of BCl-2 protein was decreased significantly, while the expression of BAX, TNF-a, cleaved caspase-3, cleaved caspase-8, cleaved caspase-9 and cleaved PARP proteins was significantly increased in the pCDNA3.1-RBM5-treated mice as compared to that in the control mice.

Conclusions: In this study, we established a novel animal model to determine RBM5 function in vivo, and concluded that RBM5 inhibited tumor growth in mice by inducing apoptosis. The study suggests that although RBM5's involvement in the death receptor-mediated apoptotic pathway is still to be investigated, RBM5-mediated growth suppression, at least in part, employs regulation of the mitochondrial apoptotic pathways.

Keywords: RBM5, Lung adenocarcinoma, Apoptosis, A549, Xenograft mice model, Attenuated Salmonella

\section{Background}

Lung cancer is the leading cause of cancer death worldwide, with over a million deaths annually [1,2]. The five-year survival rate has not improved dramatically throughout the past three decades despite innovations in diagnostic testing, surgical technique, and development of new chemotherapeutic agents $[3,4]$.

\footnotetext{
* Correspondence: wke@jlu.edu.cn

'Department of Respiratory Medicine, the Second Affiliated Hospital of Jilin University, 18 Ziqiang Street, Changchun, Jilin 130041, China

Full list of author information is available at the end of the article
}

Lung cancer histology is heterogeneous. Small cell lung carcinomas (SCLCs) account for 20\% of all lung cancers, and the remaining $80 \%$ consists of non-small cell lung carcinomas (NSCLCs) which, in turn, is further subclassified into 40\% adenocarcinoma (AC), 40\% squamous cell carcinoma (SCC) and 20\% large cell carcinoma. Delineating genetic alterations specific to each subtype of lung cancer may be the most effective way of discovering molecular markers for early detection and developing better individualized treatment.

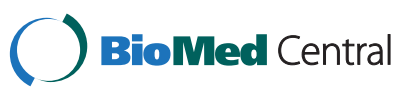


The loss of tumor suppressor gene (TSG) function is a critical step in the pathogenesis of human lung cancer. The earliest premalignant chromosomal aberration in human lung cancers is allele loss within the short arm of chromosome 3 at $3 \mathrm{p} 21.3$ [5]. This loss of heterozygosity occurs in practically all (>95\%) SCLC tumors, the majority $(>70 \%)$ of NSCLC tumors [6-8]. Deletion at chromosome $3 \mathrm{p} 21.3$ is also the most frequent genetic alteration identified in lung cancer. The region contains 19 TSGs [9], most of which demonstrate varying degrees of tumor suppressor activity (related to the control of processes such as cell differentiation, proliferation, signal transduction, and apoptosis). It has been suggested that all function together as a large, integrated, biologically functionally diverse tumor suppressor unit [7].

RNA-binding motif protein 5 (RBM5, previously referred to as g15, LUCA-15, and H37) that maps to one end of this 19-gene deletion breakpoint, is an RNAbinding protein that has the ability to modulate apoptosis and cell cycle arrest through pre-mRNA splicing of multiple target genes, such as p53 [10-17]. Overexpression of RBM5, which is also involved in the regulation of alternative splicing, showed the function of inhibiting tumor growth and reducing the metastatic potential [18-20]. In addition, multiple protein isoforms of RBM5 exist, each possessing apoptosis modulatory activity, a function consistent with tumor suppressor activity. For the inhibitory effect of RBM5 on lung cancer, in current studies, it is reported that RBM5 modulates apoptosis by regulating the alternative splicing of apoptosis-associated premRNAs, such as CASP2 and FAS/CD95 [2,21]. In Oh's study, they found RBM5 could significantly inhibit the growth of A549 non-small cell lung cancer cells by inducing apoptosis, which is associated with upregulation of the proapoptotic protein BAX, increased release of mitochondrial cytochrome $\mathrm{C}$ into the cytosol and increased activation of caspases 9 and $3[12,14,16]$. Most of the researches, however, were restricted to the cell line in vitro. In addition, in most of these approaches, the therapeutic agents could not reach the tumors in effective doses, or were distributed to unwanted sites and degraded by nucleases resulting in limited antitumor effects. Currently, there is not an effective animal model for further in vivo exploration of the function and mechanism of RBM5 on primary lung cancer. Furthermore, how to deliver a vector overexpressing RBM5 to the tumor-specific part is the key point for the study in vivo. Fortunately, the discovery that genes in bacterial vectors can be functionally transferred to mammalian cells suggests that it might be possible to use bacterial vectors as vehicles for gene therapy. Genetically modified nonpathogenic bacteria have been used as potential antitumor agents, to either elicit direct tumoricidal effects or deliver tumoricidal molecules [22]. Bioengineered attenuated strains of Salmonella enterica serovar Typhimurium have been shown to preferentially accumulate by $>1000$-fold in tumors compared with normal tissues and to become homogeneously dispersed in the tumor tissues [23]. These attenuated bacteria have been proven safe in mice, pigs and monkeys when administered intravenously [22].

In this study, we first establish an effective animal model using BALB/c nude mice treated with attenuated Salmonella as a vector carrying plasmids to explore the molecular mechanisms involved in the antitumor effect induced by RBM 5 on lung adenocarcinoma.

\section{Methods}

\section{Cell line and plasmids}

The A549 cell line was purchased from the American Tissue Type Collection (Manassas, VA, USA). Cells were grown in RPMI 1640 supplemented with 10\% fetal bovine serum as previously described [24]. Plasmids of pcDNA3.1 and pcDNA3.1-RBM5 were generously provided by Dr. Leslie Sutherland of the Research Program, Northeast Cancer Centre, Health Sciences North, Ontario, Canada.

\section{Electrotransfection of RBM5 into competent attenuated Salmonella cells}

Competent S. enterica ser. Typhimurium cells (competence) were obtained from the China-Japan Union Hospital of Jilin University in China. The competence were mixed with $1 \mu \mathrm{g}$ pcDNA3-RBM5 or $1 \mu \mathrm{g}$ pcDNA3 plasmids and cooled for 15 minutes on ice, Then the DNA could be transfected into the competence under the conditions as follows: $\mathrm{C}=25 \mu \mathrm{F}, \mathrm{PC}=200 \mathrm{ohm}, \mathrm{V}=1.25 \mathrm{kV}$ $(12.5 \mathrm{kV} / \mathrm{cm})$. Then the competence with DNA should be quickly transferred into LB Ager medium for proliferation at $37^{\circ} \mathrm{C}$. The recombinant attenuated Salmonella strains carrying plasmids were stored at $-80^{\circ} \mathrm{C}$ for later use.

\section{Establishment of A549 xenografts}

$\mathrm{BALB} / \mathrm{c}$ athymic nude male mice (nu/nu; 6 weeks) were purchased from the Institute of Zoology, Chinese Academy of Sciences, Beijing. Use of animals was in accordance with Animal Care guidelines and the protocol was approved by Jilin University Animal Care Committee. A549 cells were washed and resuspended in phosphatebuffered saline (PBS). The suspension $\left(5 \times 10^{6}\right.$ cells in $150 \mu \mathrm{L}$ per mouse) was inoculated subcutaneously into the right flanks of nude mice. The sizes of the tumors were measured using calipers starting from day 7 after cell injection until day 42 .

\section{Gene treatment}

The tumor-bearing mice were divided randomly into two groups (six mice per group) at day 21 after cell injection. 
The mice were treated at day 28 and 35 respectively through a tail mainline as follows: (a) control group (attenuated Salmonella carrying pcDNA3.1); (b) RBM5 group (attenuated Salmonella carrying pcDNA3.1RBM5) $\left(10^{8}\right.$ colony-forming units (CFU) per $50 \mu \mathrm{L}$ PBS). Then the tumors were measured using calipers every 3.5 days until day 42 . The data were plotted using the Kaplan-Meier method to analyze the tumor growth curves. The wet weight and size of the tumors were measured when the mice were sacrificed on day 42 . The tumor blocks were taken out, a part of which was fixed in formalin for hematoxylin and eosin (H\&E) staining and immunohistochemistry analysis, and another part of which was snap frozen in liquid nitrogen immediately for Western blot and terminal deoxyuridyl transferase (TdT)-mediated deoxyuridine 5'-triphosphate (dUTP) nick-end labeling (TUNEL) assay (Roche, Penzberg, Germany).

\section{TUNEL staining of apoptotic cells}

The tissue sections of xenografts from the nude mice were dewaxed and hydrated and then were subjected to TUNEL staining following the instructions provided by the manufacturer (Promega, Madison, WI, USA). Four fields were chosen randomly and analyzed. The apoptotic index was defined as follows: apoptotic index $(\%)=100 \times$ apoptotic cells/total tumor cells.

\section{H\&E and immunohistochemistry staining}

Tumors treated with recombinant Salmonella strains carrying different plasmids were H\&E stained. Immunohistochemistry analyses were carried out as described previously [25,26]. Anti-human rabbit RBM5 antibody was purchased from Abcam (Cambridge, MA, USA).

\section{Protein extraction and Western blot}

Total proteins from both tumor tissues were extracted according to the previous study [27]. Protein samples (30 ug) were then separated by SDS-PAGE and transferred onto a PVDF membrane (Millipore, Bedford, MA, USA). The primary antibodies were rabbit anti-human RBM5, Bcl-2, Bax, TNF- $\alpha$, cleaved PARP, cleaved caspase-3, cleaved caspase-8, cleaved caspase- 9 and $\beta$-actin antibodies from Abcam (Cambridge, MA, USA). The second antibody was a goat anti-rabbit IgG-HRP from Santa Cruz Biotechnology (Santa Cruz, CA, USA). Western blot was carried out as previously described [27]. The protein bands were visualized by SuperSignal West Pico Chemiluminescent Substrate (Thermo Scientific Pierce, Rockford, IL, USA), and the membranes were subjected to X-ray autoradiography. Band intensities were determined with Quantity One software (Bio-Rad, Hercules, CA, USA). Furthermore, we confirmed the reproducibility of the experiments at least three times.

\section{Statistical analysis}

Statistical comparisons were performed using analysis of variance to determine the significance of differences observed between different treatment groups. Values are expressed as mean \pm standard deviation (SD) from at least three separate experiments in which each experiment had six samples per treatment group and differences were considered significant at a $P$ value of $<0.05$.

\section{Results}

Overexpression of RBM5 inhibited tumor growth in A549 xenograft $B A L B / c$ nude mice

The potential therapeutic effect on lung adenocarcinoma xenograft growth by RBM5 was examined in tumorbearing mice treated with bacteria carrying RBM5 plasmid (Figure 1A). Dynamic tumor growth was monitored from day 7 to day 42 after injection. We showed that,

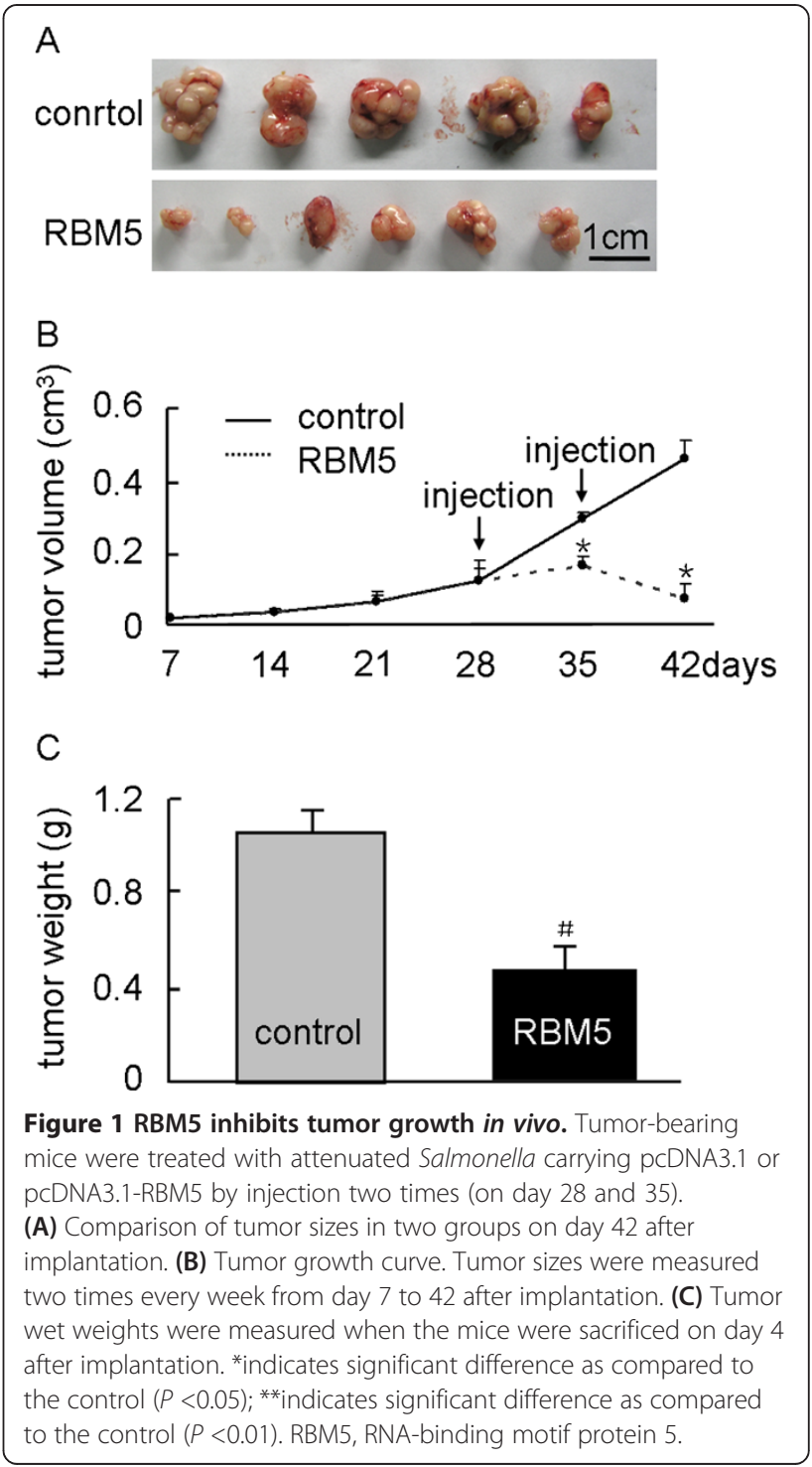


while the sizes of the tumor xenografts between RBM5 and control groups were similar before day 28, the growth of tumor xenografts in the mice treated with RBM5 retarded after day 28 (Figure 1B). In addition, the weight of the tumor xenografts from mice treated with RBM5 became significantly lighter than that in the control group at day 42 when the mice were sacrificed (Figure 1C). This result suggested that accumulative and stable expression of RBM5 in A549 xenograft BALB/c nude mice significantly retarded the tumor growth rate in vivo. Moreover, we established a novel animal model using BALB/c nude mice treated with attenuated Salmonella as a vector carrying plasmids to determine RBM5 function in vivo.

\section{Analysis of apoptosis induced by RBM5 in A549 xenograft $\mathrm{BALB} / \mathrm{C}$ nude mice}

To determine the potential mechanism of tumor growth inhibition in vivo, A549 xenograft treated with pcDNA3.1 control or pcDNA3.1-RBM5 were excised and analyzed by immunohistochemistry, H\&E, and TUNEL staining.
First, the overexpression of RBM5 was confirmed by immunohistochemistry staining. As shown in Figure 2A, RBM5 expression was significantly higher in the tumors of mice treated with pcDNA3.1-RBM5, compared to that in the tumors of mice treated with pcDNA3.1. This result suggested that the RBM5 protein was efficiently delivered and overexpressed by attenuated Salmonella in vivo.

We next employed H\&E staining to observe the histopathological performance on A549 xenografts. The result showed that there were a large number of cancer nests in the control group (Figure 2B), and the tumor tissue could survive in a good state. It could be seen, however, in the RBM5 group, that the number of cancer nests significantly decreased, and the regions of necrosis were much larger than those in the control group (Figure 2B).

To explore the contribution of cell death and tumor retardation induced by RBM5, we performed TUNEL staining analysis to detect apoptotic cells in A549 xenografts. As shown in Figure 2C, approximately more than
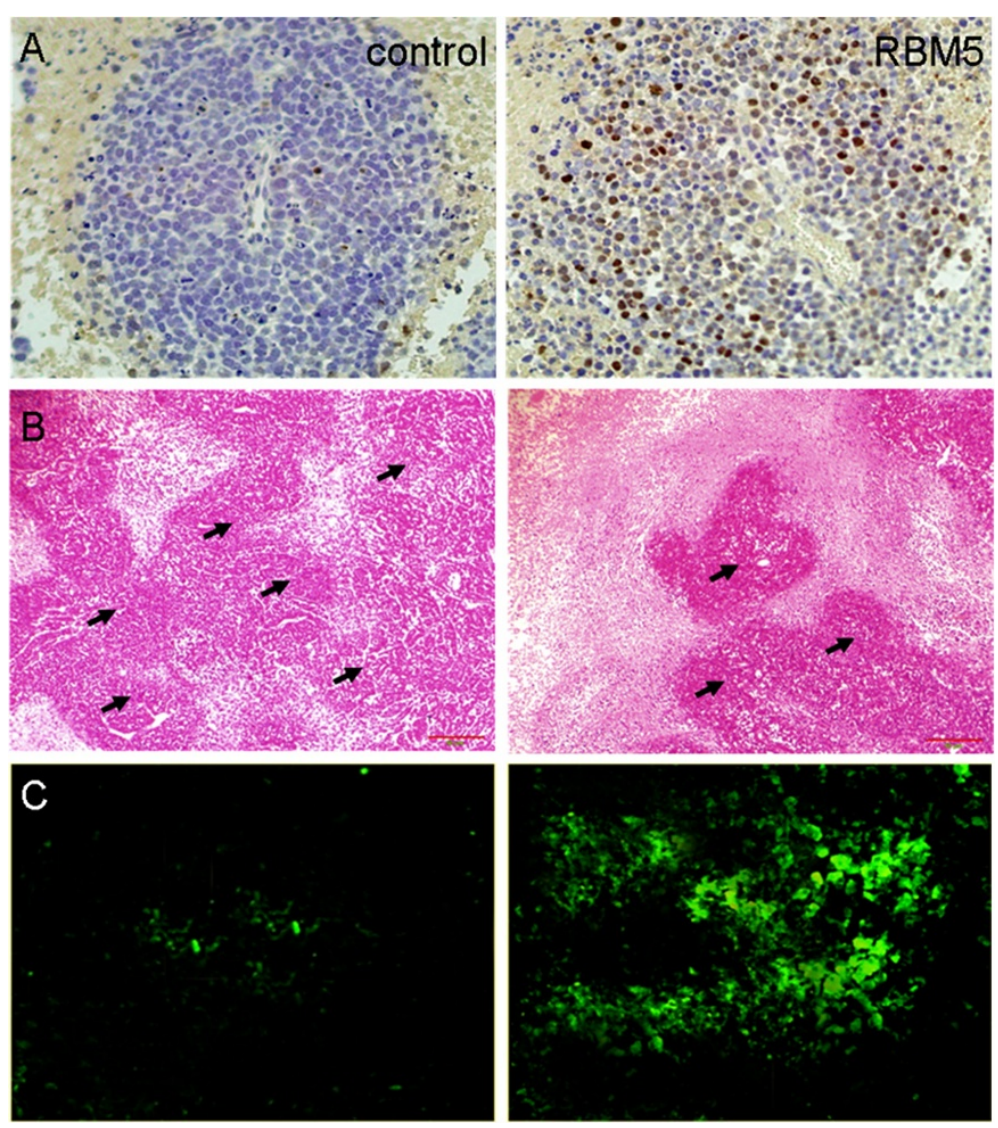

Figure 2 H\&E, immunohistochemistry and TUNEL assay. (A) Immunohistochemistry staining for RBM5 (400X). (B) H\&E staining (200X). ' $\uparrow$ ' indicates cancer nest. (C) RBM5-induced apoptosis shown by TUNEL staining (400X). Tissue sections were treated with terminal deoxynucleotidyl transferase to incorporate fluorescein-deoxyuridine 5'-triphosphate (dUTP) to nicked DNA of apoptotic cells. Apoptotic cells exhibiting a strong nuclear green fluorescence were detected by fluorescence microscopy. Adjacent light microscopic photos showed a representative area from each group showing equal cell density and fixation. RBM5, RNA-binding motif protein 5. 
ten times apoptotic cells were observed in the RBM5 group compared with those in the control group, which indicated that RBM5 inhibited A549 xenograft tumor growth by inducing apoptosis.

\section{Analysis of mitochondrial and TNF-a related apoptotic pathways in RBM5 mediated-apoptosis}

To further characterize the molecular mechanisms of the RBM5-mediated apoptotic pathways, we investigated the influence of mitochondrial apoptosis signaling and death receptors that characteristically initiate signaling. We examined the expression of some apoptosis-related genes including RBM5, Bcl-2, Bax, and TNF- $\alpha$, cleaved caspase-3, cleaved caspase-8, cleaved caspase- 9 and cleaved PARP. We observed that the expression of Bcl-2 protein was decreased significantly when RBM5 was overexpressed, while the expression of Bax, TNF- $\alpha$, cleaved caspase-3, cleaved caspase-3, cleaved caspase8 , cleaved caspase- 9 and cleaved PARP proteins were significantly increased in the RBM5 group as compared to that in the control cells (Figure 3), which suggested that, although involvement of RBM5 in the death receptor-mediated apoptotic pathway remains to be investigated in depth, RBM5- mediated growth suppression, at least in part, employs regulation of the mitochondrial apoptotic pathways.

\section{Discussion}

In recent years, lung cancer has become one of the leading causes of cancer-related death worldwide $[1,2]$. Despite innovations in diagnostic testing, surgical technique, and development of new chemotherapeutic agents, fiveyear survival rate has not improved dramatically for patients with lung cancer $[3,4]$. Therefore, it is important to understand the molecular mechanisms involved in the pathogenesis and progression of metastasis to identify novel therapeutic targets and develop effective treatment strategies.

Deletion within the lung cancer tumor suppressor region at 3p21.3 constitutes the earliest premalignant chromosomal aberration in human lung cancers. Nineteen tumor suppressor genes (TSGs) reside to this region at 3p21.3. RBM5 maps to one end of this 19-gene deletion breakpoint, which is more frequently observed in tumors of lung, breast and kidney [28], and is not included in a slightly smaller 17-gene deletion that is common to breast and renal tumors [28]. There is increasing evidence suggesting that RBM5 plays an important role in lung cancer occurrence and development, nevertheless, there are few studies reporting on RBM5 expression in lung cancer tissues and tumor cell lines. In a very small cohort of eleven specimens, it was observed that the six tumor samples with the most significantly reduced RBM5

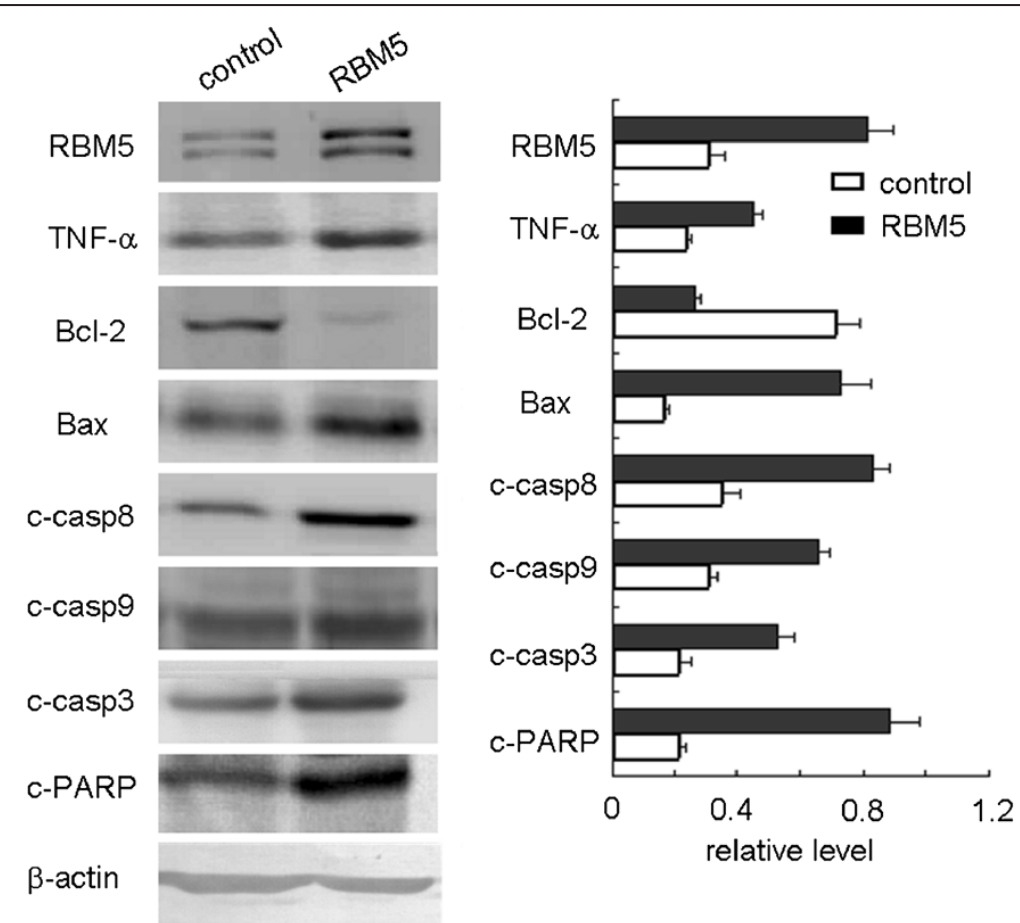

Figure 3 Differential expression of apoptosis regulatory proteins associated with RBM5 overexpression. Western blot analysis was performed to compare expression levels of various apoptosis-related proteins between the RBM5 and control groups. Among them, Bcl-2 expression was found to be decreased and RBM5, TNF-a, Bax, cleaved caspase-3, cleaved caspase-8, cleaved caspase-9 and cleaved PARP increased in the RBM5 group compared with the control. $\beta$-actin was used as a protein-loading control. RBM5, RNA-binding motif protein 5. 
mRNA levels were of the squamous type [29-31] whereas three of the nine with the less significantly reduced RBM5 mRNA levels were adenocarcinomas. The one tumor sample with no change in RBM5 mRNA expression compared to its non-tumor tissue was an adenocarcinoma, while the one tumor sample that had more RBM5 mRNA than its non-tumor tissue was a large cell carcinoma [29,31]. In our current study, we found that the expression of RBM5 mRNA and protein were both significantly reduced in 120 cases of surgically resected NSCLC compared to the adjacent normal tissues, 30 of which were lung adenocarcinoma [32]. All the above suggest the critical and specific role of RBM5 in the development of lung cancer.

This report represents the first characterization of RBM5 tumor suppressive activity and its molecular mechanism in a lung adenocarcinoma animal model. We successfully established lung adenocarcinoma animal models in vivo on the back of nude mice. We found that RBM5 significantly inhibited the growth of lung adenocarcinoma in vivo by the induction of apoptosis. In Oh's study, they also reported that RBM5 could inhibit the growth of human lung cancer A549 cells in vitro and in vivo [16]. The model they mentioned, however, was simply established using RBM5 stable transfected A549 cells to observe the growth retardation of tumor, without any metabolism for RBM5 in animals. Salmonella enterica ser. Typhi Ty21a has many of the desirable properties of a delivery vector, including targeting of multiple tumors from a distant inoculation site, selective replication within tumors, and the ability to express effector genes. Salmonella enterica ser. Typhi Ty21a has an excellent safety profile and a propensity for homing to tumors. In this study, we chose an efficient gene delivery system, attenuated S. enterica ser. Typhimurium, which could selectively target tumors and deliver the therapeutic agents into tumors while reducing the damage to normal tissue $[23,33,34]$. More recently, these bacteria have been engineered to express numerous genes, including survivin [35], MDM2 [36], Neu3 [37] and STAT3 siRNA [25] against prostate cancer in our laboratory. So this time, we first employed the attenuated $S$. enterica ser. Typhimurium to target lung tumors and directly deliver the therapeutic pcDNA3.1-RBM5 plasmids through the tail by intravenous injection.

Apoptosis is a complex, multistage process involving many genes. Among the wide spectrum of apoptosis regulatory proteins tested in our studies, the expression of proapoptotic proteins Bax, cleaved caspase-9, cleaved caspase-3, and cleaved PARP were found to be increased in RBM5-overexpressing tumor tissues, while the expression of apoptosis inhibitory protein $\mathrm{Bcl}-2$ was observed at a lower level in RBM5-overexpressing tumor tissues.
Thus, we conclude that RBM5 might induce apoptosis by triggering the mitochondrial apoptotic pathways, including caspase- 9 and caspase- 3 activations by means of increasing the expression of Bax and decreasing the expression of Bcl-2 [38]. In Oh's study, mitochondrial apoptotic pathways initiated by Bax were also observed in the A549 cells transfected with RBM5 in vitro [16]. In addition, we also noted that increased expression of TNF- $\alpha$ and cleaved caspase- 8 was associated with the RBM5 overexpression in tumor tissues. This finding, although it needs to be validated by further experiments, strongly suggested that RBM5 could initiate TNF- $\alpha$-mediated apoptosis [39], in which capase-8, caspase- 3 and PARP were activated. This potential apoptosis pathway induced by RBM5 could also be observed in human breast carcinoma MCF-7 cells [40,41] and leukemia Jurkat cells [42]. The current data suggest that although RBM5's involvement in the death receptormediated apoptotic pathway is still to be investigated in depth, RBM5-mediated growth suppression, at least in part, employs regulation of the mitochondrial apoptotic pathways.

\section{Conclusions}

In summary, we recently demonstrated that RBM5 overexpression can employ both TNF- $\alpha$-mediated apoptotic pathways and mitochondrial apoptotic pathways to suppress the growth of lung adenocarcinoma, by establishing an implant tumor model in vivo, treated with attenuated Salmonella-RBM5, a plasmids delivery system. Our studies provide further data in vivo to explore the role and molecular mechanism of RBM5 in lung cancer pathogenesis and suggest a strategy for the development of rationally designed therapeutics using RBM5 as a target.

\section{Abbreviations}

AC: Adenocarcinoma; CFU: Colony-forming unit; H\&E: Hematoxylin and eosin; NSCLCs: Non-small cell lung carcinomas; PBS: Phosphate-buffered saline; SCC: Squamous cell carcinoma; SCLCs: Small cell lung carcinomas; RBM5: RNA-binding motif protein 5; SD: Standard deviation; siRNA: Small interfering RNA; TNF-a: Tumor necrosis factor alpha; TSG: Tumor suppressor gene; TUNEL: Terminal deoxynucleotidyl transferase (TdT)-mediated deoxyuridine 5'-triphosphate (dUTP) nick-end labeling.

\section{Competing interests}

The authors declare that they have no competing interests.

\section{Authors' contributions}

CS performed all the experiments and drafted the manuscript. LZ participated in the analysis of TUNEL and H\&E staining. SW and JZ contributed to the data analysis. BY and KW have contributed to the research design, and the data collection and interpretation. KW oversaw the design of the study and was involved in the critical revision of the manuscript. All authors have read and approved the final version of the manuscript.

\section{Acknowledgements}

This work was supported by the grants from the National Natural Science Foundation of China (No. 81071919) and Provincial Natural Science Foundation of Jilin (No. 201015169) for KW and the grant from the National Natural Science 
Foundation of China for JZ (No. 30971315). We thank Dr. Leslie C. Sutherland at the Research Program, Northeast Cancer Centre, Health Sciences North in Canada for generously providing us the pcDNA3.1 and pcDNA3.1-RBM plasmids. We thank Dr. Deqi Xu at the Center for Biologics Evaluation and Research, Food and Drug Administration at Bethesda, USA for invaluable help with the attenuated Salmonella typhimurium.

\section{Author details}

${ }^{1}$ Department of Respiratory Medicine, the Second Affiliated Hospital of Jilin University, 18 Ziqiang Street, Changchun, Jilin 130041, China. ${ }^{2}$ Department of Digestive Medicine, China-Japan Union Hospital of Jilin University, 126 Xiantai Street, Changchun, Jilin 130033, China. ${ }^{3}$ Department of Pathophysiology, Norman Bethune College of Medicine of Jilin University, 18, Zhiqiang Street, Changchun, Jilin 130021, China. ${ }^{4}$ Department of Pharmacology, School of Basic Medical Sciences of Peking University, 38 Xueyuan Road, Beijing 100191, China. ${ }^{5}$ Department of Urinary Surgery, the First Affiliated Hospital of Jilin University, 71 Xinmin Street, Changchun, Jilin 130041, China.

Received: 3 February 2013 Accepted: 16 May 2013

Published: 31 May 2013

\section{References}

1. Siegel R, Naishadham D, Jemal A: Cancer statistics, 2012. CA Cancer J Clin 2012, 62:10-29.

2. Molina JR, Yang P, Cassivi SD, Schild SE, Adjei AA: Non-small cell lung cancer: epidemiology, risk factors, treatment, and survivorship. Mayo Clin Proc 2008, 83:584-594.

3. Borczuk AC, Gorenstein L, Walter KL, Assaad AA, Wang LQ, Powell CA: Nonsmall-cell lung cancer molecular signatures recapitulate lung developmental pathways. Am J Pathol 2003, 163:1949-1960.

4. Menna C, Ciccone AM, Ibrahim M, Andreetti C, D'Andrilli A, Maurizi G, Cassiano F, Cavaliere I, Venuta F, Rendina EA: Pneumonectomy: quality of life and long-term results. Minerva Chir 2012, 67:219-226.

5. Lerman MI, Minna JD, Chromosome ILC: The 630-kb lung cancer homozygous deletion region on human chromosome 3p21.3: identification and evaluation of the resident candidate tumor suppressor genes. Cancer Res 2000, 60:6116-6133.

6. Kok K, Naylor SL, Buys CH: Deletions of the short arm of chromosome 3 in solid tumors and the search for suppressor genes. Adv Cancer Res 1997, 71:27-92.

7. Ji L, Minna JD, Roth JA: 3p21.3 tumor suppressor cluster: prospects for translational applications. Future Oncol 2005, 1:79-92.

8. Wistuba II, Behrens C, Virmani AK, Mele G, Milchgrub S, Girard L, Fondon JW 3rd, Garner HR, McKay B, Latif F, Lerman MI, Lam S, Gazdar AF, Minna JD: High resolution chromosome $3 p$ allelotyping of human lung cancer and preneoplastic/preinvasive bronchial epithelium reveals multiple, discontinuous sites of $3 p$ allele loss and three regions of frequent breakpoints. Cancer Res 2000, 60:1949-1960.

9. Wei MH, Latif F, Bader S, Kashuba V, Chen JY, Duh FM, Sekido Y, Lee CC, Geil L, Kuzmin I, Zabarovsky E, Klein G, Zbar B, Minna JD, Lerman Ml: Construction of a 600-kilobase cosmid clone contig and generation of a transcriptional map surrounding the lung cancer tumor suppressor gene (TSG) locus on human chromosome 3p21.3: progress toward the isolation of a lung cancer TSG. Cancer Res 1996, 56:1487-1492.

10. Sutherland LC, Edwards SE, Cable HC, Poirier GG, Miller BA, Cooper CS, Williams GT: LUCA-15-encoded sequence variants regulate CD95mediated apoptosis. Oncogene 2000, 19:3774-3781.

11. Rintala-Maki ND, Sutherland LC: LUCA-15/RBM5, a putative tumour suppressor, enhances multiple receptor-initiated death signals. Apoptosis 2004, 9:475-484.

12. Mourtada-Maarabouni M, Sutherland LC, Williams GT: Candidate tumour suppressor LUCA-15 can regulate multiple apoptotic pathways. Apoptosis 2002, 7:421-432

13. Mourtada-Maarabouni M, Sutherland LC, Meredith JM, Williams GT: Simultaneous acceleration of the cell cycle and suppression of apoptosis by splice variant delta- 6 of the candidate tumour suppressor LUCA-15/RBM5. Genes Cells 2003, 8:109-119.

14. Sutherland LC, Lerman M, Williams GT, Miller BA: LUCA-15 suppresses CD95-mediated apoptosis in Jurkat T cells. Oncogene 2001, 20:2713-2719.
15. Sutherland KD, Lindeman GJ, Choong DY, Wittlin S, Brentzell L, Phillips W, Campbell IG, Visvader JE: Differential hypermethylation of SOCS genes in ovarian and breast carcinomas. Oncogene 2004, 23:7726-7733.

16. Oh JJ, Razfar A, Delgado I, Reed RA, Malkina A, Boctor B, Slamon DJ: 3p21.3 tumor suppressor gene H37/Luca15/RBM5 inhibits growth of human lung cancer cells through cell cycle arrest and apoptosis. Cancer Res 2006, 66:3419-3427.

17. Li P, Wang K, Zhang J, Zhao L, Liang H, Shao C, Sutherland LC: The 3p21.3 tumor suppressor RBM5 resensitizes cisplatin-resistant human non-small cell lung cancer cells to cisplatin. Cancer Epidemiol 2012, 36:481-489.

18. Oh JJ, Taschereau EO, Koegel AK, Ginther CL, Rotow JK, Isfahani KZ, Slamon DJ: RBM5/H37 tumor suppressor, located at the lung cancer hot spot 3p21.3, alters expression of genes involved in metastasis. Lung Cancer 2010, 70:253-262.

19. Akhtar MJ, Ahamed M, Khan MA, Alrokayan SA, Ahmad I, Kumar S: Cytotoxicity and apoptosis induction by nanoscale talc particles from two different geographical regions in human lung epithelial cells. Environ Toxicol 2012. doi:10.1002/tox.21766. [Epub ahead of print]

20. Niu ZY, Jin WX, Zhang LB, Li XL: Tumor suppressor RBM5 directly interacts with the DExD/H-box protein DHX15 and stimulates its helicase activity. FEBS Lett 2012, 586:977-983.

21. Fushimi K, Ray P, Kar A, Wang L, Sutherland LC, Wu JY: Up-regulation of the proapoptotic caspase 2 splicing isoform by a candidate tumor suppressor, RBM5. Proc Natl Acad Sci USA 2008, 105:15708-15713.

22. Zhao M, Yang M, Li XM, Jiang $P$, Baranov E, Li S, Xu M, Penman S, Hoffman RM: Tumor-targeting bacterial therapy with amino acid auxotrophs of GFPexpressing Salmonella typhimurium. Proc Natl Acad Sci USA 2005, 102:755-760.

23. Low KB, Ittensohn M, Le T, Platt J, Sodi S, Amoss M, Ash O, Carmichael E, Chakraborty A, Fischer J, Lin SL, Luo X, Miller SI, Zheng L, King I, Pawelek JM, Bermudes D: Lipid A mutant Salmonella with suppressed virulence and TNFalpha induction retain tumor-targeting in vivo. Nat Biotechnol 1999, 17:37-41.

24. Mu YM, Oba K, Yanase T, Ito T, Ashida K, Goto K, Morinaga H, Ikuyama S, Takayanagi R, Nawata H: Human pituitary tumor transforming gene (hPTTG) inhibits human lung cancer A549 cell growth through activation of p21(WAF1)/CIP1. Endocr J 2003, 50:771-781.

25. Zhang L, Gao L, Li Y, Lin G, Shao Y, Ji K, Yu H, Hu J, Kalvakolanu DV, Kopecko DJ, Zhao X, Xu DQ: Effects of plasmid-based Stat3-specific short hairpin RNA and GRIM-19 on PC-3M tumor cell growth. Clin Cancer Res 2008, 14:559-568.

26. Gao L, Zhang L, Hu J, Li F, Shao Y, Zhao D, Kalvakolanu DV, Kopecko DJ, Zhao X, Xu DQ: Down-regulation of signal transducer and activator of transcription 3 expression using vector-based small interfering RNAs suppresses growth of human prostate tumor in vivo. Clin Cancer Res 2005, 11:6333-6341.

27. Lin GM, Zhao LJ, Yin F, Lan RF, Li LB, Zhang XM, Zhang H, Yang BX: TCF3 inhibits F9 embryonal carcinoma growth by the down-regulation of Oct4. Oncol Rep 2011, 26:893-899.

28. Lerman Ml, Minna JD: The 630-kb lung cancer homozygous deletion region on human chromosome 3p21.3: identification and evaluation of the resident candidate tumor suppressor genes. The International Lung Cancer Chromosome 3p21.3 Tumor Suppressor Gene Consortium. Cancer Res 2000, 60:6116-6133.

29. Angeloni $D$, ter Elst $A$, Wei MH, van der Veen AY, Braga EA, Klimov EA, Timmer T, Korobeinikova L, Lerman MI, Buys CH: Analysis of a new homozygous deletion in the tumor suppressor region at $3 p 12.3$ reveals two novel intronic noncoding RNA genes. Genes Chromosomes Cancer 2006, 45:676-691.

30. Ramaswamy S, Ross KN, Lander ES, Golub TR: A molecular signature of metastasis in primary solid tumors. Nat Genet 2003, 33:49-54.

31. Oh JJ, West AR, Fishbein MC, Slamon DJ: A candidate tumor suppressor gene, H37, from the human lung cancer tumor suppressor locus 3p21.3. Cancer Res 2002, 62:3207-3213.

32. Liang $H$, Zhang J, Shao C, Zhao L, Xu W, Sutherland LC, Wang K. Differential expression of RBM5, EGFR and KRAS mRNA and protein in non-small cell lung cancer tissues. J Exp Clin Cancer Res 2012, 31:36.

33. King I, Itterson M, Bermudes D: Tumor-targeted Salmonella typhimurium overexpressing cytosine deaminase: a novel, tumor-selective therapy. Methods Mol Biol 2009, 542:649-659.

34. Gardlik R, Fruehauf JH: Bacterial vectors and delivery systems in cancer therapy. IDrugs 2010, 13:701-706. 
35. Shao Y, Liu Y, Shao C, Hu J, Li X, Li F, Zhang L, Zhao D, Sun L, Zhao X, Kopecko DJ, Kalvakolanu DV, Li Y, Xu DQ: Enhanced tumor suppression in vitro and in vivo by co-expression of survivin-specific siRNA and wildtype p53 protein. Cancer Gene Ther 2010, 17:844-854.

36. Ji K, Wang B, Shao YT, Zhang L, Liu YN, Shao C, Li XJ, Li X, Hu JD, Zhao XJ, XU DQ, Li Y, Cai L: Synergistic suppression of prostatic cancer cells by coexpression of both murine double minute 2 small interfering RNA and wild-type p53 gene in vitro and in vivo. J Pharmacol Exp Ther 2011, 338:173-183,

37. Li X, Zhang L, Shao Y, Liang Z, Shao C, Wang B, Guo B, Li N, Zhao X, Li Y, Xu D: Effects of a human plasma membrane-associated sialidase siRNA on prostate cancer invasion. Biochem Biophys Res Commun 2011, 416:270-276.

38. Israels LG, Israels ED: Apoptosis. Stem Cells 1999, 17:306-313.

39. Ashkenazi A, Dixit VM: Death receptors: signaling and modulation. Science 1998, 281:1305-1308.

40. Rintala-Maki ND, Abrasonis V, Burd M, Sutherland LC: Genetic instability of RBM5/LUCA-15/H37 in MCF-7 breast carcinoma sublines may affect susceptibility to apoptosis. Cell Biochem Funct 2004, 22:307-313.

41. Davis FA, Haque MS, Ulatowski TG, Towson JC: Asymmetric oxidation of ester and amide enolates using new (camphorylsulfonyl)oxaziridines. J Org Chem 1986, 51:2402-2404.

42. Dudley E, Hornung F, Zheng L, Scherer D, Ballard D, Lenardo M: NF-kappaB regulates Fas/APO-1/CD95- and TCR-mediated apoptosis of T lymphocytes. Eur J Immunol 1999, 29:878-886.

doi:10.1186/1477-7819-11-123

Cite this article as: Shao et al:: Tumor suppressor gene RBM5 delivered by attenuated Salmonella inhibits lung adenocarcinoma through diverse apoptotic signaling pathways. World Journal of Surgical Oncology 2013 11:123.

\section{Submit your next manuscript to BioMed Central and take full advantage of:}

- Convenient online submission

- Thorough peer review

- No space constraints or color figure charges

- Immediate publication on acceptance

- Inclusion in PubMed, CAS, Scopus and Google Scholar

- Research which is freely available for redistribution 